\title{
The aftereffects of ventriloquism: Patterns of spatial generalization
}

\author{
PAUL BERTELSON \\ Université Libre de Bruxelles, Brussels, Belgium \\ and \\ ILJA FRISSEN, JEAN VROOMEN, and BÉATRICE DE GELDER \\ Tilburg University, Tilburg, The Netherlands
}

\begin{abstract}
We examined how visual recalibration of apparent sound location obtained at a particular location generalizes to untrained locations. Participants pointed toward the origin of tone bursts scattered along the azimuth, before and after repeated exposure to bursts in one particular location, synchronized with point flashes of light a constant distance to their left/right. Adapter tones were presented straight ahead in Experiment 1, and in the left or right periphery in Experiment 2. With both arrangements, different generalization patterns were obtained on the visual distractor's side of the auditory adapter and on the opposite side. On the distractor side, recalibration generalized following a descending gradient; practically no generalization was observed on the other side. This dependence of generalization patterns on the direction of the discordance imposed during adaptation has not been reported before, perhaps because the experimental designs in use did not allow its observation.
\end{abstract}

A substantial part of behavioral research on crossmodal interaction has focused on relations between auditory and visual processing of related inputs. As for the equally welldocumented case of the interactions between proprioception and vision, studied mostly with prismatic rearrangement, the main approach has been based on the imposition of experimental conflict between the information provided in the two modalities concerning one aspect, or dimension, of the inputs (Howard, 1982; Welch, 1978).

The most extensively studied auditory-visual conflict is the one concerning spatial location. When auditory and visual stimuli such as tone bursts and light flashes are presented synchronously but in different locations, the apparent location of the auditory stimulus is typically shifted in the direction of the visual stimulus (Bermant \& Welch, 1976; Bertelson \& Aschersleben, 1998; Bertelson, Pavani, Làdavas, Vroomen, \& de Gelder, 2000; Bertelson \& Radeau, 1981; Bertelson, Vroomen, \& de Gelder, 1997; Bertelson, Vroomen, de Gelder, \& Driver, 2000; Hairston et al., 2003; Klemm, 1909; Radeau, 1992; Radeau \& Bertelson, 1987; Thomas, 1941; Vroomen, Bertelson, \& de Gelder, 2001; for reviews see Bertelson, 1999; Bertelson \& de Gelder, 2004; Welch, 1999; Welch \& Warren, 1980). This "visual bias of perceived sound location" generally represents only a fraction of the sound-flash

The present work was supported by the Belgian National Fund for Collective Fundamental Research (Contract 10759/2001 to Régine Kolinsky and P.B.). Constructive criticisms by three anonymous reviewers and editor Mark Pitt are gratefully acknowledged. Correspondence should be addressed to P. Bertelson, Laboratoire de Psychologie Expérimentale, Université Libre de Bruxelles, 50 Av. F. D. Roosevelt, B-1050 Brussels, Belgium (e-mail: pbrtlsn@ulb.ac.be). distance. It can nevertheless be sufficient to bring the perceived discrepancy below the detection threshold, which could explain the illusion created by performing ventriloquists that the speech they produce without visible articulation comes from a synchronously agitated dummy. The term ventriloquism has, for that reason, come to be used to designate collectively all manifestations of auditoryvisual spatial interaction (Bertelson, 1999; Howard \& Templeton, 1966). When the converse effect, the auditory bias of visual location, was measured as well, it was small but nevertheless reached significance in some studies (Bertelson \& Radeau, 1981; Radeau \& Bertelson, 1987; Warren, Welch, \& McCarthy, 1981; see Radeau, 1985, for a negative result).

Apart from the immediate, or online, effects represented by crossmodal biases, ventriloquism also manifests itself through offline aftereffects (AEs), by which the apparent location of test sounds, presented unimodally after a period of exposure to spatially incongruent sound-flash pairs, is displaced, in relation to preexposure tests, in the direction of the preceding visual competitors (Canon, 1970; Frissen, Vroomen, de Gelder, \& Bertelson, 2003, 2005; Lewald, 2002; Radeau, 1973, 1992; Radeau \& Bertelson, 1969, 1974, 1976, 1977, 1978; Recanzone, 1998; Zwiers, Van Opstal, \& Paige, 2003). The converse AEs, postexposure shifts of visual localization in the opposite direction - that is, toward the auditory competitors - can also be obtained, but these are generally of smaller amplitude (Radeau \& Bertelson, 1969, 1974, 1976; see Lewald, 2002, Experiment 1, for a partial replication).

The occurrence of AEs has generally been attributed to a process of perceptual learning, whereby the correspondences between stimuli and resulting percepts are recali- 
brated, in both modalities, or at least in one of them, in a way that reduces the existing incongruence. Such recalibrations should play a role in the development, or in the later maintenance, of crossmodal coordination (see, e.g., de Gelder \& Bertelson, 2003; Held, 1961; Welch, 1978).

Another reason for being interested in AEs consists in the information that they can provide regarding the extent of the interactions caused by intermodal conflicts. Measuring AEs at several stimulus values after exposure to conflict at a particular one can tell us whether interactions involve only the stimuli present during exposure, or also a range of stimuli along the discordance dimension. This research strategy was first put forward by Bedford (1989). Taking the case of the conflict between the seen and felt locations of a body part (the traditional object of prism adaptation studies), she considered the possibility that recalibrations might affect whole perceptual dimensions rather than just the particular stimulus (or stimuli) involved in the conflict. She measured finger pointing to visual targets before and after a period spent pointing to prismatically displaced targets. On each exposure trial, the participant received feedback (lighting of an LED) whenever the responding finger entered a critical area around the target. In the main experiment, recalibration achieved at one of three different azimuthal locations was found to generalize entirely across a whole range of test locations ( $52.5^{\circ}$ on either side of straight ahead). Thus, AEs from adaptation carried out at a particular location generalized entirely across a $105^{\circ}$ range.

Bedford's (1989) finding was consistent with those from earlier studies carried out with different main objectives, but in which there were similarly no significant differences between AEs observed at trained and at untrained locations (Baily, 1972; Harris, 1963; Hay, Langdon, \& Pick, 1971). Quite different generalization patterns were obtained in two later studies, however. Ghahramani, Wolpert, and Jordan (1996) had participants point at visual targets at various locations in a two-dimensional horizontal area, with remappings imposed, as in Bedford's experiments, by visual feedback. To judge from the vector field graphs in the paper, postexposure shifts tended to be largest at the exposure location and to go down with distance from that location. Field, Shipley, and Cunningham (1999) exposed participants to a task in which they tried to intercept with an unseen finger a falling ball that was visible, for a variable segment of its trajectory, through laterally displacing prisms. With vision of the ball through a sufficiently long segment, generalization followed a typical diminishing gradient pattern, with a peak AE at exposure location and rapid reductions on both sides of that location. No convincing explanation has been proposed for those diverging data, and in fact the tasks that were used differed on so many dimensions that no easily testable hypothesis presents itself.

The generalization paradigm has rarely been applied to AEs of ventriloquism. Four recent studies have considered generalization along the dimension of sound frequency, again with rather diverging results. In two studies (Lewald,
2002; Recanzone, 1998), no generalization was observed over distances in the order of two octaves, whereas total or near total generalization was reported over up to four octaves in the other two (Frissen et al., 2003, 2005). Reasons for these differences are currently being tested, and they will not be discussed here.

In the present study, we examine, for the first time in the ventriloquism literature, generalization across space. Participants pointed to the apparent location of sound bursts delivered in several azimuthal locations, before and after exposure to a series of identical sound bursts in one particular location, each accompanied by a synchronous point flash of light, a constant angular distance to either its left or its right. Adaptation was conducted with the sound in the participant's median plane in Experiment 1, and in two peripheral locations, respectively in the left and the right half spaces, in Experiment 2. The focus of the study was the kind of spatial generalization pattern that would obtain in these situations.

Since the study was focused on generalization, its feasibility was conditional on one's obtaining the usual basic adaptation at the exposure location. To obtain it, we simply resorted to procedures that in our earlier work, as well as that of colleagues, had proved capable of bringing it about. It must on the other hand be clear that we were not trying to answer any particular question regarding the conditions of occurrence of the basic effect. For instance, no particular measures were taken to ensure that the participants attended to the visual distractors, earlier results having shown that such attention was not necessary to obtain either visual bias (Bertelson, Vroomen, et al., 2000) or auditory AEs (Frissen et al., 2003). Had the expected adaptation not occurred, we would simply have had to reconsider our procedures.

Three patterns of generalization were considered possible: (1) no generalization-that is, recalibration restricted to the locus of adaptation; (2) uniform generalization across the azimuth (as found by Bedford, 1989); or (3) generalization following a decreasing gradient on both sides of adaptation locus (as found by Field et al., 1999).

\section{GENERAL METHOD}

The testing was carried out in a dark, semireverberant and soundproof booth, $4.6 \mathrm{~m}$ long, $2.4 \mathrm{~m}$ wide, and $2.2 \mathrm{~m}$ high. Participants sat in front of a table with their heads restrained by a fixed chinrest at $\sim 40 \mathrm{~cm}$ above the tabletop. The setup involved seven display units for presentation of auditory and visual stimuli, and an array of push buttons to be used by participants in auditory localization tests. The display units, which were hidden behind a black, acoustically transparent cloth, were arranged in a semicircular array on the vertical plane of the chinrest and at a $42-\mathrm{cm}$ radial distance from it, one straight ahead $\left(0^{\circ}\right)$ and the others at $17.5^{\circ}, 35^{\circ}$, and $52.5^{\circ}$ to the left and right of straight ahead. Each of them consisted of a loudspeaker (Philips, $30-\mathrm{W}$ wide frequency Box $410, \varnothing=9 \mathrm{~cm})$ with a red LED $(\varnothing=$ $1 \mathrm{~cm}$ ) over its center. The pushbuttons, a total of 108, were arranged on the table top, along another semicircular array, at $1^{\circ}$ intervals, and $5 \mathrm{~cm}$ in front of the display units (thus at $37-\mathrm{cm}$ horizontal distance from the chinrest).

The auditory stimuli, used in pre- and posttests as well as in bimodal exposure trials, consisted of a single 200 -msec burst of a 
750-Hz pure tone, with 5-msec linear rise/fall envelopes, presented at $66 \mathrm{~dB}(\mathrm{~A})$. Speakers had a characteristic that was flat within $12 \mathrm{~dB}$ between .3 and $15 \mathrm{kHz}$, with approximately 5 -dB/octave roll-off. Reverberation times (measured in the booth with all the experimental equipment in place) were $330 \mathrm{msec}$ at $500 \mathrm{~Hz}$ and $270 \mathrm{msec}$ at $1000 \mathrm{~Hz}$, meaning that for our $750-\mathrm{Hz}$ tone it was presumably less than $330 \mathrm{msec}$. The flashes also lasted $200 \mathrm{msec}$, and their luminance was set at $28 \mathrm{~cd} / \mathrm{m}^{2}$, measured from a continuous light at a distance of $1 \mathrm{~m}$. When delivered (on bimodal exposure trials only), they were clearly visible through the occluding cloth.

\section{EXPERIMENT 1 Adaptation in Central Location}

In this experiment, adaptation was carried out with the sounds coming from straight ahead and with the discordant visual stimuli on either of the next display units, $17.5^{\circ}$ to the left or to the right. Its effects were measured through pre- and postexposure localization tests with auditory targets at seven equidistant locations: straight ahead $\left(0^{\circ}\right)$, and $17.5^{\circ}, 35^{\circ}$, and $52.5^{\circ}$ left and right.

\section{Method}

Participants. Sixteen students from Tilburg University (age, 18 $25 ; 12$ were female), all naive as to the purpose of the experiment and with normal hearing and normal or corrected-to-normal vision, participated in two sessions each.

Procedure. Each of the two sessions was run throughout with the distractor flashes either left or right of the sounds, in balanced order. A session began with 98 auditory pretests, 14 from each of the seven loudspeakers, in randomized order. These were initiated by the participant's pressing a button located in the median plane, $20 \mathrm{~cm}$ in front of her/him, and the sound followed after $500 \mathrm{msec}$. This procedure ensures a constant starting position for all pointing movements. Instructions were that one should always press the pushbutton closest to the apparent direction of the sound, and no stress whatever was put on response speed. The session continued with seven adaptation posttest blocks. Each of these blocks involved 60 bimodal exposure trials, followed by 14 posttests. On a bimodal exposure trial, the $200-\mathrm{msec}$ sound was delivered in the median loudspeaker simultaneously with a flash in the next display unit, $17.5^{\circ}$ to the left or to the right, depending on the session. Participants were told to look at the location in which flashes were delivered. It follows from the preceding description that exposure trials were always identical for the seven blocks of each session. They followed each other at 1 -sec intervals, so that each exposure phase lasted just above $1 \mathrm{~min}$ (exactly $60.2 \mathrm{sec}$ ). There was a $3.5-\mathrm{sec}$ interval between the end of each exposure phase and the start of the following set of posttests. The 14 posttests, 2 from each loudspeaker, in randomized order, were self-paced just as the pretests, so that the total duration of a posttest phase varied between participants. It typically lasted 40-60 sec. The following exposure phase began when the participant again pressed the median button. For the 98 pretests, typical durations were 5-7 $\mathrm{min}$.

\section{Results}

Responses were filtered for outliers by discarding, separately for each sound test location, values lying more than 2.5 standard deviations from the mean. These represented $1.3 \%$ of the data. AEs were then calculated by subtracting mean reported locations on pretests (14 values per participant and per sound location) from those on posttests (2 values per participant and sound location for each block $\times 7$ blocks, making 14 values again). AEs were counted as positive when they went toward the visual distractor.

Figure 1 shows, separately for each direction of discordance (visual distractor to the left vs. to the right), mean AEs measured at the different locations. Two main points of interest emerge. First, both generalization functions have a peak in the vicinity of the adaptation location (here, straight ahead). In the figure, the two peaks occur in fact at different points, at the straight ahead location for leftward discordance, but at the next location to the right of straight ahead for rightward discordance. This aspect of the results should actually not detain us, for a paired $t$ test applied to participants' individual peak locations fell short of significance $[t(15)=1.20, p=.25]$. Mean peak locations (leftward discordance, $11.9^{\circ}$ left; rightward, $1.1^{\circ}$ right) were thus not significantly different. Also, none of the mean locations were significantly different from the median location [leftward discordance, $t(15)=-1.72$, $p=.11$; rightward, $t(15)=0.15, p=.88]$.

Second, the two curves follow asymmetrical courses on the two sides of their respective peaks, and this asymmetry varies with direction of discordance. For leftward discordance, substantial AEs are found for tests carried out in the left half space, and practically none in the right half space. The opposite asymmetry occurs for the rightward discordance. Thus generalization occurs mainly, if not only, in the direction in which sounds were attracted during the preceding exposure.

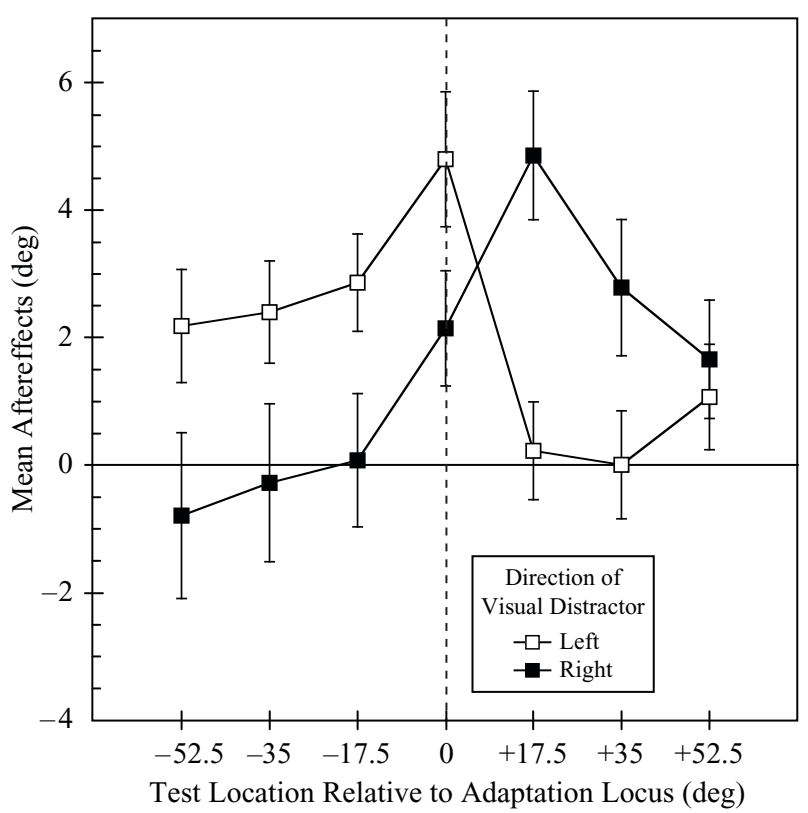

Figure 1. Experiment 1: Adaptation in central location. Mean aftereffects (and standard errors) are presented as functions of test location. During exposure, the sounds were always presented at $0^{\circ}$ (dotted line), and the visual distractors, at $17.5^{\circ}$ to the left or to the right depending on the discordance direction. 
The data were first submitted to a 2 (discordance direction [DD]: leftward vs. rightward) $\times 7$ (test location) repeated measures ANOVA. The main effect of DD was nonsignificant $(F<1)$, but its interaction with test location was highly significant $[F(6,90)=7.58, p<.0001]$, reflecting the opposite asymmetries of the generalization patterns obtained under the two DDs. The main effect of test location was also significant $[F(6,90)=3.32, p<$ $.01]$, but given the strong interaction with $\mathrm{DD}$, this fact has no meaningful implication.

To further examine the dependence of the results on $\mathrm{DD}$, the AEs were considered and analyzed in terms of their being measured on the side of the auditory adapter on which the visual distractor was delivered during exposure (henceforth distractor side), or on the opposite side (nondistractor side). In Figure 2, the mean AEs obtained at generalization (noncentral) locations are shown as functions of their distance from display center, on respectively the distractor side (on the left) and the nondistractor side (on the right). The AEs obtained in the central location, which belong to neither category, were not included in the new analysis. They are shown in gray in the figure.

The new ANOVA was a 2 (test side: distractor vs. nondistractor) $\times 2$ (DD) $\times 3$ (distance) repeated measures design. Distances were entered in absolute values $\left(17.5^{\circ}\right.$, $35.0^{\circ}$, and $\left.52.5^{\circ}\right)$. The main effects of test side $[F(1,15)=$ $10.13, p<.01]$ and distance $[F(2,30)=3.40, p<.05]$ were significant, but that of DD $(F<1)$ was not. Among interactions, those between distance and DD $[F(2,30)=$

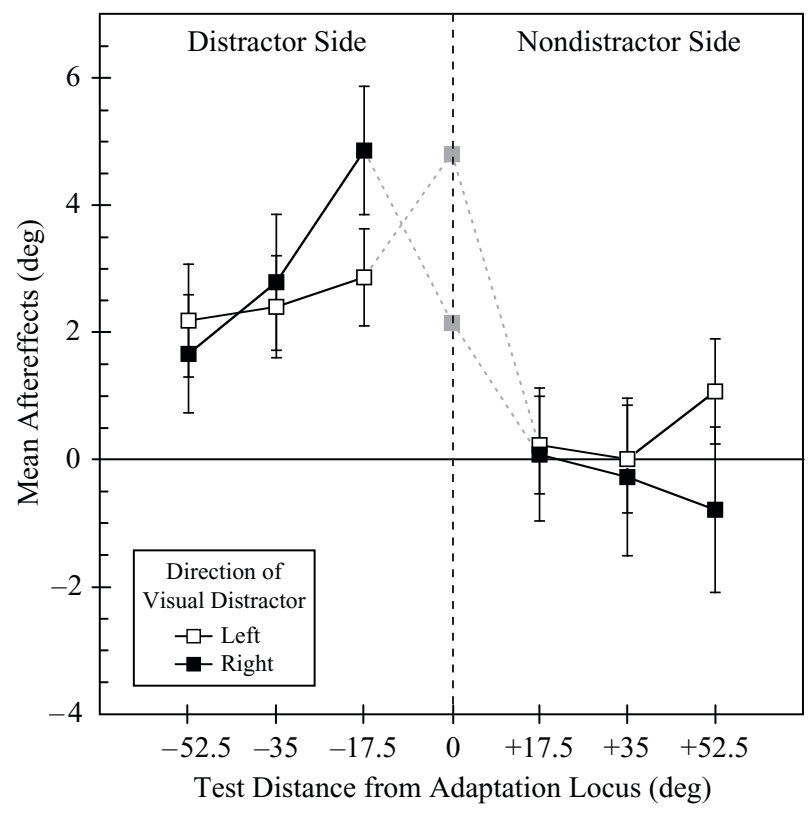

Figure 2. Experiment 1: Adaptation in central location. Same data as in Figure 1, replotted as functions of distance from the central adaptation locus, measured along the distractor side of that locus (left part) or on the nondistractor side (right part). Aftereffects at the adaptation locus, which belong to neither category and were not included in the ANOVA, are shown in gray.
$3.71, p<.05]$ and test side $[F(2,30)=4.10, p<.05]$ were both significant, whereas that between test side and DD was not, nor was the second-order interaction (both $F_{\mathrm{S}}<1$ ). The distance $\times$ test side interaction corresponds to the fact, visible on the figure, that the effect of distance is smaller on the nondistractor side than on the distractor side. The distance $\times$ DD interaction reflects the fact, also visible on the figure, that the effect of distance is present on both test sides for rightward discordance, and smaller (or even inverted, on the nondistractor side) for leftward discordance.

\section{Discussion}

As expected, exposure to the present form of auditoryvisual conflict produced significant auditory AEs at, or in the vicinity of, exposure location. The condition was thus obtained for examining spatial generalization. Here, a surprising pattern occurred. Whatever the direction of discordance, different patterns of generalization occurred on the two sides of adapter sound location. In the two cases, substantial generalization was found on the distractor side, and practically none on the nondistractor side. On the distractor side, generalization followed a gradient that diminished with increasing distance from center, hence presumably from adaptation location. On the nondistractor side, no generalization was found, meaning either that none occurred, or possibly one that went down with distance so rapidly that it had vanished at the first location at which it was examined, $17.5^{\circ}$ from adaptation location.

\section{EXPERIMENT 2} Adaptation in the Periphery

In Experiment 1, adaptation was induced in the median plane. In this second experiment, spatial generalization was examined for adaptation at more peripheral locations, with the target sound $35^{\circ}$ on either side of center. This arrangement allowed the consideration of test locations across a wider angle $\left(87.5^{\circ}\right)$, from adaptation location toward center and beyond. Our main purpose was to test the generality of the findings of Experiment 1 with respect to the generalization gradients, in particular their dependence on DD.

\section{Method}

Participants. Fourteen new students from the same pool (age, $18-27 ; 8$ were female), all naive again as to the purpose of the experiment and with normal hearing and normal or corrected-tonormal vision, participated in four sessions each.

Procedure. One of the four sessions was devoted to each combination of adapter sound location (left vs. right periphery) and DD (visual distractor to left vs. right of adapter sound). Just as in Experiment 1 , each session began with 98 auditory pretests, 14 from each of the seven loudspeakers, in randomized order, and continued with seven adaptation posttest blocks. Each of these blocks consisted of 60 bimodal exposure trials, with the sound, depending on the session, at $35^{\circ}$ to the left or right of straight ahead, and the flash from the next display unit, $17.5^{\circ}$ to its left or right, followed again immediately by 14 auditory posttests, 2 from each of the seven loudspeakers, in randomized order. Other aspects of the procedure, like the gaze orientation, pointing instructions, and the keypressing initiation of test trials, were the same as in Experiment 1. 
As mentioned above, generalization was, for each adaptation location, measured on one side at five locations extending from the adapter to the center and then beyond, but on the other side at the single remaining location only. Given our interest in the shape of generalization gradients, the data from these single locations were for each condition excluded from the analysis. The corresponding test trials can thus be considered as fillers.

\section{Results}

Outlying responses, amounting to $1.2 \%$ of the data, were discarded again.

Mean AEs per sound test location, computed again by subtracting mean pointed locations on pretests from those on posttests, are shown, separately for the four conditions, in Figure 3. The left panel shows the data for adaptation in the left half space, and the right panel, for the right half space.

AEs again have clear maxima at adaptation locations, and go down when measured at other locations. On the other hand, both the peaks and the generalization gradients depend on DD. This dependence is strongest for adaptation in the left half space. When the visual distractor is delivered on the right side of the adapter sound, a substantial peak (more than $6^{\circ}$ ) obtains at adaptation location and AEs go down with increasing distance from peak location, following a quasi-monotonic gradient. With the distractor on the opposite side (i.e., to the left), the starting peak is practically at zero level, and AEs become increasingly negative at the next three locations, before going back to starting level. In the right half space, a similar (though less accentuated) pattern is obtained. AEs are higher at both the adaptation location and the next two locations for the condition with the distractor toward the center (i.e., to the left). With the distractor away from center (i.e., to the right), the starting peak is also lower and a final rebound (similar to the one obtained in the left half space) occurs again at the more distant locations.

The differences in peak values between conditions with the distractor on the center side of the sound and on the other side were tested with paired $t$ tests. The difference was significant for adaptation in the left half space $[t(13)=3.59, p<.01]$, and not for adaptation in the right half space $(t<1)$.

In order to better illustrate the role of DD, the data were regrouped in Figure 4, with generalization on the distractor side and on the nondistractor side in separate panels. The similarity of the curves in each panel is very apparent, as are the differences between the panels.

The AEs were submitted to a 2 (DD: leftward vs. rightward) $\times 2$ (test side: distractor vs. nondistractor) $\times$ 6 (distance) repeated measures ANOVA. Distance was again entered in absolute values $\left(0^{\circ}, 17.5^{\circ}, 35^{\circ}, 52.5^{\circ}, 70^{\circ}\right.$, and $87.5^{\circ}$ ). The main effect of test side was significant $[F(1,13)=14.64, p<.01]$, and its interaction with DD was not $(F<1)$. The main effect of distance was highly significant $[F(5,65)=22.9, p<.001]$, but this factor's interactions with DD $[F(5,65)=4.01, p<.01]$ and with test side $[F(5,65)=4.93, p<.01]$ were also significant. The main effect of DD fell narrowly short of significance $[F(1,13)=3.98, p=.067]$, and the second-order interaction $[F(5,65)=1.36, p=.25]$ was also nonsignificant.

The effects of distance were further explored through trend analyses carried out separately on the distractor and on the nondistractor side. On the distractor side, the linear component was highly significant $[F(1,13)=30.9, p<$ $.0001]$, and all higher order ones were nonsignificant. The linear trend's interaction with DD (left vs. right) was nonsignificant $(F<1)$. On the nondistractor side, there was

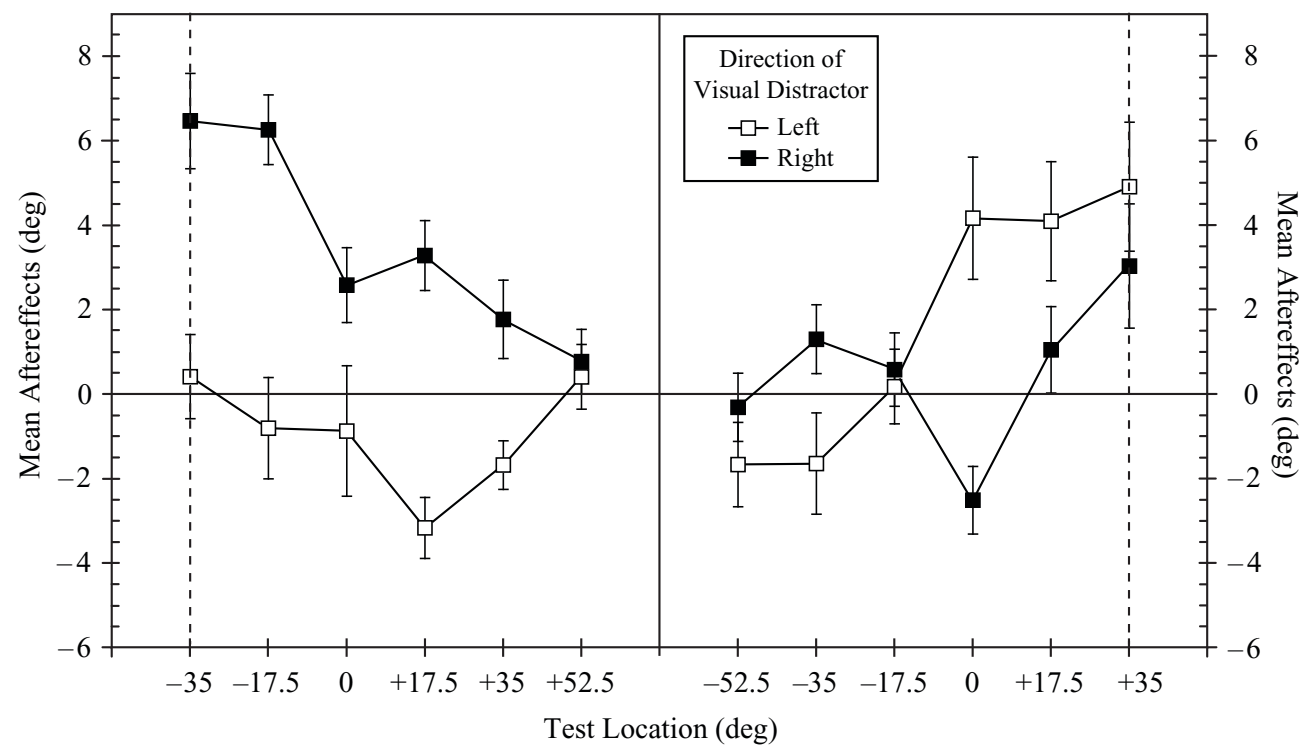

Figure 3. Experiment 2: Adaptation in the periphery. Mean aftereffects (and standard errors) are presented as functions of test location. During exposure, sounds (dotted vertical lines) were presented at $35^{\circ}$ left (left panel) or right (right panel). 


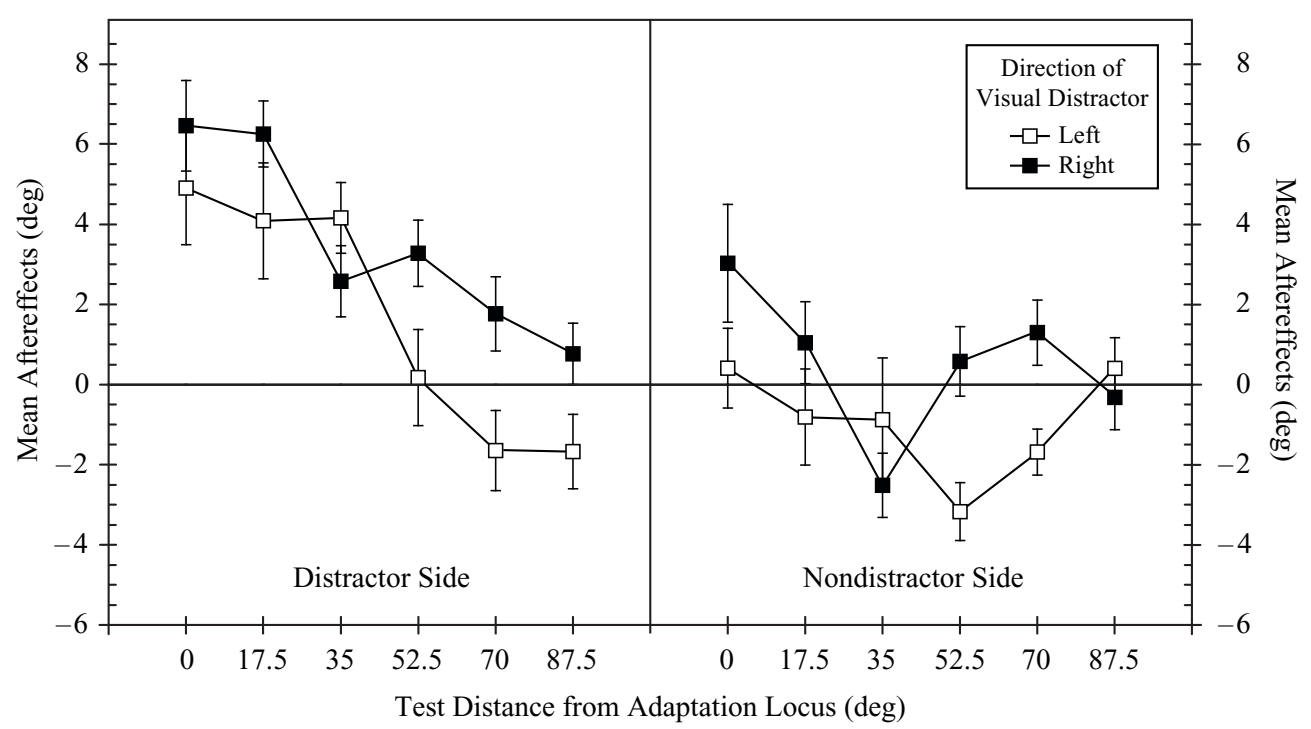

Figure 4. Experiment 2: Adaptation in the periphery. Same data as in Figure 3, replotted as functions of absolute distance from the adaptation locus. Left panel: adaptation toward center; right panel: adaptation away from center.

no significant linear component $(F<1)$, only a quadratic one $[F(1,13)=15.1, p<.002]$. The latter reflects the rebound in both functions. Its interaction with DD was also nonsignificant $(F<1)$.

\section{Discussion}

Several of the results of Experiment 2 call for comments.

After exposure at each of the two adaptation loci, more generalization occurred when the visual distractor had been presented on the central side of the auditory adapter than on the lateral side. Since the generalization was in both cases measured at locations extending from the adaptation locus toward center and beyond, the effect means that, just as in Experiment 1, generalization was stronger in the direction of the former visual distractor than in the opposite direction.

The larger set of locations over which generalization was now measured provided a picture of generalization more complete than the one obtained in Experiment 1. On the distractor side, that picture is clearly one of a gradient diminishing monotonically with distance from adaptation locus. On the other hand, in the two conditions with testing on the nondistractor side, AEs rebounded upward at the largest distances from adaptation location. These rebounds were probably responsible for the significant nonlinear trends found specifically in these conditions. There is for the time being no obvious explanation for that particular aspect of the results.

Finally, in the present experiment, DD affected not only the pattern of generalization, but also the adaptation occurring at the adaptation locations themselves. At both these locations, the $\mathrm{AE}$ was larger in the condition with the distractor on the central side. This difference might suggest that part of the present influence of DD on generalization may be a consequence of the adaptation occurring at the adaptation locus, higher adaptation peaks producing more generalization. This relation could of course not account for the whole of the DD effect, since in Experiment 1 the two DDs created different generalization patterns from the same peak adaptations. Moreover, the effect on adaptation peak reached significance at only one of the two adaptation loci (in the left half space). No strong conclusions can be drawn concerning this particular issue until the data have received a more general confirmation.

\section{GENERAL DISCUSSION}

As presented in the introduction, the main purpose of this study was to determine how recalibration of the apparent direction of a sound obtained through ventriloquism in a particular location generalizes to untrained locations. Three patterns were considered possible: uniform extension across locations, decreasing gradients, or no generalization. The uniform generalization pattern was clearly not found. AEs always peaked around the location at which sounds had been delivered during exposure (straight ahead in Experiment 1 and $35^{\circ}$ left or right in Experiment 2), and went down when measured away from that location.

The novel, and completely unexpected, outcome of the study is, however, that different patterns were observed on the side of the visual distractor and the opposite side. This discordance direction (DD) effect was most clearly demonstrated in Experiment 1, with the auditory adapter straight ahead and the visual distractor on either its left or its right. In both cases, substantial generalization, going down with increasing distance from the adaptation locus, 
was found on the distractor side, and no, or very little, generalization was found on the nondistractor side. The same effect of DD was observed in Experiment 2. With the auditory adapters in the periphery, generalization could be measured only at locations extending toward the center and beyond. Clear generalization occurred only in the conditions in which the distractor had itself been located on the same central side of the adapter sound. In these conditions, a clear monotonically decreasing gradient was obtained. When the distractor had been presented on the noncentral side, practically no generalization was observed.

It has been asked whether the present effects- the recalibration and its pattern of generalization - really resulted from the preceding exposure to auditory-visual discordance, and not simply from gaze deviation toward the visual distractor during exposure. If the apparent direction of sound sources moved along with the direction of gaze, an effect mimicking the visual bias of sound location could be produced and be subsequently consolidated as an AE. This proposal actually runs into several difficulties.

First, if the manifestations of ventriloquism were simple consequences of fixation on the visual distractor, they would occur equally with synchronized and desynchronized bimodal presentations. Actually, desynchronizing visual and auditory inputs has been shown to eliminate (Bertelson \& Aschersleben, 1998) or at least strongly reduce (Bertelson et al., 1997; Choe, Welch, Gilford, \& Juola, 1975; Radeau \& Bertelson, 1987; Thomas, 1941; Warren et al., 1981) the visual bias of perceived auditory location. And in another study (Radeau \& Bertelson, 1977), AEs consequent on exposure to congruent auditory and visual data (in this case, auditory speech and the sight of the talker's face) presented in separate locations were also reduced significantly by desynchronization. Another result that is similarly inconsistent with the gaze direction explanation was reported by Recanzone (1998). In one of his experiments, this author exposed 3 participants to series of synchronized auditory clicks and visual flashes delivered either in separate locations ( $8^{\circ}$ apart) or in the same location, in the left or the right half space. He obtained strong auditory AEs in the first condition, and none in the latter one. Had gaze direction been the critical factor, the same adaptations would obviously have occurred in the two conditions.

A second problem for the proposed gaze direction explanation comes from studies directly focused on the effects of eye deviation on sound localization. Lewald $(1997,1998)$ has in a succession of papers shown convincingly that the most typical immediate effect is a shift away from fixation, thus opposite the usual direction of visual bias. Regarding recalibration, the same author has recently reported a replication of Recanzone's (1998) comparison between AEs of exposure to sound-flash pairs in respectively separate (here $20^{\circ}$ apart) and aligned locations (Lewald, 2002, Experiments 1 and 2). With spatially separate pairs, the perceived sound location shifted as usual toward the visual distractors, but with spatially aligned ones, there was a significant tendency for shifts to go instead in the direction opposite the deviation of adapters from straight ahead. For instance, both auditory and visual stimuli were localized more to the left after right half space presentations than after left half space presentations. The inverse pattern, postexposure shifts toward the focus of gaze, has been reported, however, in one condition of a study by Weerts and Thurlow (1971). The participants' localization of trains of auditory clicks was measured before and after a period spent monitoring for occasional flashes of an LED situated $20^{\circ}$ to the left or to the right of their heads' median plane. Postexposure localizations were shifted, in comparison with baseline data obtained with the LED, just like the auditory source, in the median plane, by a small (about $2^{\circ}$ ) but significant amount in the direction of the preceding deviation. This result was not replicated by Radeau and Bertelson (1977) for exposure to auditory and visual speech in separate locations. In a control condition, the participants monitored (for occasional flashes) an LED located $20^{\circ}$ to one side of straight ahead while listening to speech from a frontal source, and no AEs whatever were obtained.

It is thus unlikely that in our experiments, the orientation of the gaze toward the visual distractors made any substantial contribution to the generation of the AEs observed at the adaptation locus. If that factor went in the direction found by Lewald (2002), its effect may actually have been to counteract the contribution from spatial recalibration.

Another critical comment that we have received was that the DD effect might have been created by sound reverberations occurring in our experimental booth and might not occur if the experiments were run in a fully anechoic room. The problem with that notion is that in each of our exposure conditions, the total acoustic input, the main component plus its eventual reverberations, was the same irrespective of the side, left or right, on which the visual distractor was delivered. So, how could presence or absence of reverberations explain a directional effect on sound localization?

So it appears that the DD effect really tells us something about the spatial extension of auditory recalibration. This recalibration would apply differently in the two halves of the discordance dimension, which, given that the gaze was in all probability directed toward the visual distractor for most of the exposure phases, means the two half visual fields. In the half toward which the target sound is moved, the shift would extend to other locations along the dimension, with a strength that decreases with distance. In the other half, there would be no generalization (or, as already mentioned, one that decreases too fast to be still visible at the smallest $17.5^{\circ}$ distance considered in our study).

An obvious question is whether this generalization pattern reflects a constraint specific to auditory localization, or one that applies also in other modalities. As we have seen in the introduction, the only other case of spatial recalibration for which patterns of generalization have been examined is the visual recalibration of proprioception. 
Unfortunately, nearly all the relevant studies have been carried out with a single DD, so that the only available source of information about possible DD effects was the comparison between AEs obtained at corresponding eccentricities in the two half spaces. These AEs could have been influenced, beyond DD, by specific characteristics of the locations at which they had been measured, such as, for instance, local susceptibility to recalibration. For example, in Field et al.'s (1999) experiments with the falling ball task, which were run with rightward prismatic displacement only, symmetrical gradients occurred on the two sides of exposure location. This result might suggest that DD was not an effective factor in that situation. There is, however, a possibility that a real DD effect, producing in this case stronger generalization in the right half space, happened to be counterbalanced by a higher susceptibility to recalibration of points in the left half space. An apparently opposite kind of result was obtained by Ghahramani et al. (1996), who found some effects that, although the authors did not mention it, might have been related to DD. In one of their conditions, which involved remapping of pointing toward the body along the sagittal axis, generalization (as judged from their Figure 7B) occurred only on the remapping side of the adaptation locus. However, in the absence of data for remapping in the opposite direction, the result could here also reflect some local differences in susceptibility to recalibration as well as a DD effect. The only way to effectively rule out contamination of generalization results by local factors is thus to carry out all recalibrations, as was done in the present study, in two opposite directions. The effects of changing DD could then be measured in exactly the same locations. Until such controls have been applied, our question regarding DD effects in cases other than auditory recalibration receives no answer.

\section{REFERENCES}

BAILY, J. (1972). Adaptation to prisms: Do proprioceptive changes mediate adapted behavior with ballistic arm movements? Quarterly Journal of Experimental Psychology, 24, 8-20.

BEDFORD, F. L. (1989). Constraints on learning new mappings between perceptual dimensions. Journal of Experimental Psychology: Human Perception \& Performance, 15, 232-248.

Bermant, R. I., \& Welch, R. B. (1976). Effect of degree of separation of visual-auditory stimulus and eye position upon spatial interaction of vision and audition. Perceptual \& Motor Skills, 42, 487-493.

Bertelson, P. (1999). Ventriloquism: A case of cross-modal perceptual grouping. In G. Aschersleben, T. Bachmann, \& J. Müsseler (Eds.), Cognitive contributions to the perception of spatial and temporal events (pp. 347-362). Amsterdam: Elsevier.

Bertelson, P., \& Aschersleben, G. (1998). Automatic visual bias of perceived auditory location. Psychonomic Bulletin \& Review, 5, 482 489.

Bertelson, P., \& de Gelder, B. (2004). The psychology of multimodal perception. In C. Spence \& J. Driver (Eds.), Crossmodal space and crossmodal attention (pp. 141-177). Oxford: Oxford University Press.

Bertelson, P., Pavani, F., Làdavas, E., Vroomen, J., \& de Gelder, B. (2000). Ventriloquism in patients with unilateral visual neglect. $\mathrm{Neu}$ ropsychologia, 38, 1634-1642.

Bertelson, P., \& Radeau, M. (1981). Cross-modal bias and perceptual fusion with auditory-visual spatial discordance. Perception \& Psychophysics, 29, 578-584.
Bertelson, P., Vroomen, J., \& de Gelder, B. (1997). Auditory-visual interaction in voice localization and in bimodal speech recognition: The effects of desynchronization. In C. Benoit \& R. Campbell (Eds.), ESCA workshop on audio-visual speech processing, cognitive and computational approaches (pp. 97-100). Rhodes.

Bertelson, P., Vroomen, J., De Gelder, B., \& Driver, J. (2000). The ventriloquist effect does not depend on the direction of deliberate visual attention. Perception \& Psychophysics, 62, 321-332.

CANON, L. K. (1970). Intermodality inconsistency of input and directed attention as determinants of the nature of adaptation. Journal of Experimental Psychology, 84, 141-147.

Choe, C. S., Welch, R. B., Gilford, R. M., \& Juola, J. F. (1975). The "ventriloquist effect": Visual dominance or response bias? Perception \& Psychophysics, 18, 55-60.

de Gelder, B., \& Bertelson, P. (2003). Multisensory integration, perception and ecological validity. Trends in Cognitive Sciences, 7 , 460-467.

Field, D. P., Shipley, T. F., \& Cunningham, D. W. (1999). Prism adaptation to dynamic events. Perception \& Psychophysics, 61, 161-176.

Frissen, I., Vroomen, J., de Gelder, B., \& Bertelson, P. (2003). The aftereffects of ventriloquism: Are they sound-frequency specific? Acta Psychologica, 113, 315-327.

Frissen, I., Vroomen, J., de Gelder, B., \& Bertelson, P. (2005). The aftereffects of ventriloquism: Generalization across soundfrequencies. Acta Psychologica, 118, 93-100.

Ghahramani, Z., Wolpert, D. M., \& Jordan, M. I. (1996). Generalization to local remappings of the visuomotor coordinate transformation. Journal of Neuroscience, 16, 7085-7096.

Hairston, W. D., Wallace, M. T., Vaughan, J. W., Stein, B. E., NorRIS, J. L., \& SCHIRILlo, J. A. (2003). Visual localization ability influences cross-modal bias. Journal of Cognitive Neuroscience, 15, 20-29.

HARRIS, C. S. (1963). Adaptation to displaced vision: Visual, motor, or proprioceptive change? Science, 140, 812-813.

HaY, J. C., LAngdon, B., \& Pick, H. L. (1971). Spatial parameters of eye-hand adaptation to optical distortion. Journal of Experimental Psychology, 91, 11-17.

Held, R. (1961). Sensory deprivation: Facts in search of a theory. Exposure-history as a factor in maintaining stability of perception and co-ordination. Journal of Nervous \& Mental Disease, 132, 26-32.

HowARD, I. P. (1982). Human visual orientation. New York: Wiley.

Howard, I. P., \& Templeton, W. B. (1966). Human spatial orientation. London: Wiley.

KLEMM, O. (1909). Lokalisation von Sinneneindrücken bei disparaten Nebenreizen [Localization of sensory impressions with disparate distractors]. Psychologische Studien, 5, 73-161.

LEWALD, J. (1997). Eye-position effects on directional hearing. Behavioural Brain Research, 87, 35-48.

LEWALD, J. (1998). The effect of gaze eccentricity on perceived sound direction and its relation to visual localization. Hearing Research, 115, 206-216

LEWALD, J. (2002). Rapid adaptation to auditory-visual spatial disparity. Learning \& Memory, 9, 268-278.

RADEAU, M. (1973). The locus of adaptation to auditory-visual conflict. Perception, 2, 327-332.

RadeAU, M. (1985). Signal intensity, task context, and auditory-visual interactions. Perception, 14, 571-577.

RadeAu, M. (1992). Cognitive impenetrability in auditory-visual interaction. In J. Alegria, D. Holender, J. Morais, \& M. Radeau (Eds.), Analytic approaches to human cognition (pp. 41-55). Amsterdam: Elsevier.

Radeau, M., \& Bertelson, P. (1969). Adaptation à un déplacement prismatique sur la base de stimulations exafférentes en conflit. Psychologica Belgica, 9, 133-140.

Radeau, M., \& Bertelson, P. (1974). The after-effects of ventriloquism. Quarterly Journal of Experimental Psychology, 26, 63-71.

RAdeAu, M., \& Bertelson, P. (1976). The effect of a textured visual field on modality dominance in a ventriloquism situation. Perception \& Psychophysics, 20, 227-235.

Radeau, M., \& Bertelson, P. (1977). Adaptation to auditory-visual discordance and ventriloquism in semirealistic situations. Perception \& Psychophysics, 22, 137-146. 
Radeau, M., \& Bertelson, P. (1978). Cognitive factors and adaptation to auditory-visual discordance. Perception \& Psychophysics, 23, 341-343.

Radeau, M., \& Bertelson, P. (1987). Auditory-visual interaction and the timing of inputs: Thomas (1941) revisited. Psychological Research, 49, 17-22.

Recanzone, G. H. (1998). Rapidly induced auditory plasticity: The ventriloquism aftereffect. Proceedings of the National Academy of Sciences, 95, 869-875.

Thomas, G. J. (1941). Experimental study of the influence of vision on sound localization. Journal of Experimental Psychology, 28, 163-177.

Vroomen, J., Bertelson, P., \& DE Gelder, B. (2001). The ventriloquist effect does not depend on the direction of automatic visual attention. Perception \& Psychophysics, 63, 651-659.

Warren, D. H., Welch, R. B., \& McCARThy, T. J. (1981). The role of visual-auditory "compellingness" in the ventriloquism effect: Implications for transitivity among the spatial senses. Perception $\&$ Psychophysics, 30, 557-564.
Weerts, T. C., \& Thurlow, W. R. (1971). The effects of eye position and expectation on sound localization. Perception \& Psychophysics, 9, 35-39.

WeLCH, R. B. (1978). Perceptual modification: Adapting to altered sensory environments. New York: Academic Press.

Welch, R. B. (1999). Meaning, attention, and the unity assumption in the intersensory bias of spatial and temporal perceptions. In G. Aschersleben, T. Bachmann, \& J. Müsseler (Eds.), Cognitive contributions to the perception of spatial and temporal events (pp. 371387). Amsterdam: Elsevier.

Welch, R. B., \& Warren, D. H. (1980). Immediate perceptual response to intersensory discrepancy. Psychological Bulletin, 88, 638-667.

Zwiers, M. P., Van Opstal, A. J., \& Paige, G. D. (2003). Plasticity in human sound localization induced by compressed spatial vision. Nature Neuroscience, 6, 175-181.

(Manuscript received July 14, 2004;

revision accepted for publication May 31, 2005.) 